\title{
Posttraumatic stress disorder (PTSD) and complex PTSD (CPTSD) as per ICD-11 proposals: A population study in Israel
}

\author{
Menachem Ben-Ezra ${ }^{1 *}$ (D) | Thanos Karatzias PhD ${ }^{2,3 *}$ | Philip Hyland $\mathrm{PhD}^{4 *}$ \\ Chris R. Brewin PhD ${ }^{5}$ | Marylene Cloitre PhD ${ }^{6,7}$ | Jonathan I.Bisson MD ${ }^{8}$ | \\ Neil P. Roberts PhD ${ }^{9} \quad$ Brigitte Lueger-Schuster PhD ${ }^{10}$ | Mark Shevlin PhD ${ }^{11 *}$
}

${ }^{1}$ School of Social Work, Ariel University, Ariel, Israel

${ }^{2}$ School of Health and Social Care, Edinburgh Napier University, Edinburgh, United Kingdom

${ }^{3}$ Rivers Centre for Traumatic Stress, NHS Lothian, Edinburgh, United Kingdom

${ }^{4}$ School of Business, National College of Ireland, Dublin, Ireland

${ }^{5}$ Clinical Educational \& Health Psychology, University College London, London, United Kingdom

${ }^{6}$ National Center for PTSD, Veterans Affairs Palo Alto Health Care System, Palo Alto, CA, USA

${ }^{7}$ School of Medicine, New York University, New York, NY, USA

${ }^{8}$ School of Medicine, Cardiff University, Cardiff, United Kingdom

${ }^{9}$ Psychology and Counselling Directorate, Cardiff \& Vale University Health Board, Cardiff, United Kingdom

${ }^{10}$ Faculty of Psychology, University of Vienna,

Vienna, Austria

${ }^{11}$ School of Psychology and Psychology

Research Institute, Ulster University, Magee

Campus, Derry, Northern Ireland, United

Kingdom

Correspondence

Menachem Ben-Ezra, School of Social Work, Ariel University, Ariel Science Park, Ariel 40700 , Israel.

Email:m.shevlin@ulster.ac.uk

*M.B., T.K., P.H., and M.S. contributed equally to this paper and share equal responsibility.
Background: The current study sought to advance the existing literature by providing the first assessment of the factorial and discriminant validity of the ICD-11 proposals for posttraumatic stress disorder (PTSD) and complex PTSD (CPTSD) in a nationwide level.

Methods: A nationally representative sample from Israel ( $n=1,003)$ using a disorder-specific measure (ITQ; International Trauma Questionnaire) in order to assess PTSD and CPTSD along with the Life Events Checklist and the World Health Organization Well-Being Index.

Results: Estimated prevalence rates of PTSD and CPTSD were 9.0 and 2.6\%, respectively. The structural analyses indicated that PTSD and disturbances in self-organization symptom clusters were multidimensional, but not necessarily hierarchical, in nature and there were distinct classes that were consistent with PTSD and CPTSD.

Conclusions: These results partially support the factorial validity and strongly support the discriminant validity of the ICD-11 proposals for PTSD and CPTSD in a nationally representative sample using a disorder-specific measure; findings also supported the international applicability of these diagnoses. Further research is required to determine the prevalence rates of PTSD and CPTSD in national representative samples across different countries and explore the predictive utility of different types of traumatic life events on PTSD and CPTSD.

KEYWORDS

CFA, CPTSD, ICD-11, PTSD

\section{1 | INTRODUCTION}

Two "sibling disorders" have been proposed for the 11th version of the International Classification of Diseases (ICD-11): posttraumatic stress disorder (PTSD) and complex PTSD (CPTSD) (Karatzias et al., 2017). The organizing principles for the ICD-11 revisions are that diagnoses should be consistent with clinicians' mental health taxonomies, limited in the number of symptoms, and based on distinctions important for management and treatment (Karatzias et al., 2017). The ICD-11 model of PTSD includes six symptoms measuring three clusters (each cluster is composed of two symptoms): (1) re-experiencing of the trauma in the present (Re), (2) avoidance of traumatic reminders (Av), and (3) a persistent sense of threat that is manifested by increased arousal and hypervigilance (Th). These symptoms are intended to define PTSD as a response characterized by some degree of fear or horror directly related to a specific traumatic event or series of events.

CPTSD is conceptualized as a broader diagnosis recognizing the pervasive psychological damage that may result from sustained, repeated, and multiple forms of traumatic exposures (e.g., childhood abuse, domestic violence; political imprisonment) (Brewin et al., 2017). 
The ICD-11 model of CPTSD is composed of six symptom clusters: three are shared with PTSD and three that are collectively referred to as "disturbances in self-organization" (DSO): affective dysregulation (AD), negative self-concept (NSC), and disturbed relationships (DR). The AD symptoms reflect difficulties with regulating emotions, manifesting in terms of hyperactivation (e.g., heightened emotional reactivity, anger outbursts) or hypoactivation (e.g., feeling emotionally numb or dissociated) of emotional states. Problematic emotion regulation strategies, both hyper- and hypoactivation, are commonly observed consequences of sustained traumatic exposure (Dvir, Ford, Hill, \& Frazier, 2014). The NSC symptoms reflect extreme negative selfevaluations and persistent negative views of the self. An extensive literature attests to the frequency with which negative self-perceptions are observed following traumatic exposure, particularly traumatic exposures of an interpersonal nature (Badour \& Adams, 2015). The DR symptoms reflect difficulties with developing and sustaining interpersonal relationships (e.g., feeling distant from others, having difficulty maintaining relationships). Social withdrawal, isolation, and disconnection from others are commonly observed consequences of exposure to early life, interpersonal traumas (Walsh, Fortier, \& DiLillo, 2010). Although CPTSD has been substantially investigated in terms of its structure (three PTSD symptom clusters and three DSO clusters), the selection of symptom indicators for each DSO cluster has yet to be finalized (Karatzias et al., 2016, 2017).

Efforts to date to test the construct validity of ICD-11 PTSD and CPTSD have predominantly utilized factor-analytic (confirmatory factor analysis [CFA]) and mixture-modeling (latent class/profile analysis [LCA/LPA]) approaches. There have been nine CFA studies that have assessed the latent symptom structure of CPTSD, utilizing samples from different nationalities (e.g., Germany, United Kingdom, Denmark, Timor-Leste) and different traumatic backgrounds (e.g., childhood institutional abuse, sexual assault, refugee status, polytraumatization) (Cloitre, Garvert, Brewin, Bryant, \& Maercker, 2013; Hyland et al., 2017a, 2017b; Hyland, Brewin, \& Maercker, 2017d; Karatzias et al., 2017; Knefel \& Lueger-Schuster, 2013; Nickerson et al., 2016; Shevlin et al., 2017; Tay et al., 2017; Tay, Rees, Chen, Kareth, \& Silove, 2015). In every single study the CFA results provided empirical support for the distinction between PTSD and DSO, in line with the ICD-11 proposals, with results indicating two viable structural representations: (1) a correlated six-factor, first-order model (Re, Av, Th, AD, NSC, and $\mathrm{DR}$ ), and (2) a correlated two-factor, second-order model whereby a second-order PTSD factor explains the covariation between the $\mathrm{Re}, \mathrm{Av}$, and Th factors, and a second-order DSO factor explains the covariation between the AD, NSC, and DR factors.

Ten LCA/LPA studies have been conducted to date, again utilizing samples of varied nationalities and traumatic backgrounds. In eight of these studies, qualitatively distinct classes reflecting the distinction between PTSD and CPTSD symptom profiles were identified (Cloitre et al., 2013; Cloitre, Garvert, Weiss, Carlson, \& Bryant, 2014; Elklit, Hyland, \& Shevlin, 2014; Karatzias et al., 2016, 2017; Knefel, Garvert, Cloitre, \& Lueger-Schuster, 2015; Murphy, Elklit, Dokkedahl, \& Shevlin, 2016; Perkonigg et al., 2016; Sachser, Keller, \& Goldbeck, 2017; Wolf et al., 2015). In one study, the results suggested that the observed classes were quantitatively, rather than qualitatively, distinct (Wolf et al., 2015). In other words, the observed symptom patterns did not reflect the qualitative distinction between PTSD and CPTSD, rather individuals seemed to be responding at different levels of intensity (e.g., low, medium, high) to the same underlying disorder.

The existing literature has also identified a number of psychosocial and demographic factors that predict PTSD and CPTSD responses. In line with the wider trauma literature, females appear to be approximately twice as likely as males to experience PTSD and CPTSD (Hyland et al., 2017a; Karatzias et al., 2017). Elevated risk of PTSD has been associated with single-incident, adult traumatic exposure (Cloitre et al., 2013), elevated anxiety-based symptoms (Hyland et al., 2017a, 2017b; Knefel \& Lueger-Schuster, 2013), and repeated exposure to the same trauma (Glück, Knefel, Tran, \& Lueger-Schuster, 2016; Hyland et al., 2017b). CPTSD has been associated with chronic childhood trauma (Cloitre et al., 2013), childhood sexual abuse (Hyland et al., 2017b; Karatzias et al., 2017), and exposure to multiple forms of childhood traumatization (Cloitre et al., 2013; Hyland et al., 2017b; Karatzias et al., 2017), increased functional impairment (Cloitre et al., 2013; Hyland et al., 2017c; Karatzias et al., 2017), greater psychiatric burden (Elklit, Hyland, \& Shevlin, 2014; Perkonigg et al., 2016), elevated depressive symptoms (Hyland et al., 2017a, 2017c), distress intolerance (Hyland et al., 2017a, 2017c), being unmarried (Karatzias et al., 2017), and being unemployed (Hyland et al., 2017b; Karatzias et al., 2017).

To date, the extant research evidence appears to support the construct validity of the ICD-11's proposals for PTSD and CPTSD. However, there have been some salient limitations associated with all previous studies. First, many studies have used ad hoc items from other measurement instruments as proxy indicators of the symptoms proposed by the ICD-11 working group. This unavoidable limitation has recently been addressed with the development of the International Trauma Questionnaire (ITQ: Cloitre, Roberts, Bisson, \& Brewin, 2015), a self-report measure specifically designed to capture the ICD-11 symptoms of PTSD and DSO. Second, to date there has been no study that has examined the latent symptom structure of CPTSD (via CFA methods), or the distinguishability of PTSD and CPTSD symptom presentations (via LCA/LPA methods), among a nationally representative sample of trauma-exposed adults. As such, while the factorial validity and the discriminant validity of PTSD and CPTSD is well established among clinical populations, it is unclear if these constructs are supported among community populations. Third, in all prior CFA studies the $A D$ component of DSO has been modeled as a unidimensional construct, despite the fact that it is explicitly composed of two related dimensions of hyperactivation and hypoactivation. The unidimensional representation of the $A D$ factor in prior studies has followed from guidelines set forth by the ICD-11 working group (Maercker et al., 2013), but given the ultimate intention to represent the $A D$ factor using one hyperactivation symptom and one hypoactivation symptom, there is a need to formally test whether these dimensions are distinct (reflecting two correlated dimensions) or can be considered as two congeneric measures of a single underlying dimension.

Using a Hebrew version of the newly developed ITQ (Cloitre et al., 2015), the current study aimed to: (1) estimate the prevalence of PTSD and CPTSD in a large, nationally representative sample of 
trauma-exposed adults in Israel, (2) assess if the PTSD and CPTSD diagnoses are associated with demographic and trauma variables, (3) use CFA to test the factorial validity of CPTSD, acknowledging the delineation between the hyper- and hypoactivation symptoms of AD, and (4) use LCA to test the discriminant validity of ICD-11 PTSD and CPTSD by determining if there are separate classes of individuals identifiable by symptom profiles consistent with the distinction between PTSD and CPTSD.

\section{2 | METHODS}

\section{1 | Participants and procedures}

The study sample comprised 1,003 trauma-exposed Israeli adults (response rate $=31 \%$ ). Within the Israeli (Middle East) context, recent armed conflicts along with terror attacks put almost the entire Israeli population under direct or potential threat to life, corresponding with exposure to a traumatic event. The study used an internet panel of about 130,000 Israelis that adheres to the Israeli Bureau of Statistics in key demographic factors that represent the general population (Bodas, Siman-Tov, Kreitler, \& Peleg, 2017). In order to maintain its representativeness, the panel is undergoing dynamic changes according to changes in the Israeli census based on data from the Israel Bureau of Statistics. From this panel, a sample of 1,003 Israelis was selected using stratified and random sampling methods in order to obtain a sample that is a close approximation to the general population. Potential participants were invited to participate in the study via e-mail. Each participant signed an electronic informed consent form before accessing the questionnaire. Eligibility to participate in the study included being over the age of 18, and being fluent in Hebrew. The mean age of the sample was 40.6 years $(S D=14.5$; range $18-70)$ and there were more women (51.7\%) than men. All the participants were born in Israel. The majority (82.3\%) reported living in urban areas, and $70.5 \%$ reported being in a committed relationship. The average number of children was 1.8 $(S D=1.7$; range $0-11)$. The majority reported being employed either in a full-time (61.8\%) or part-time (20.9\%) job. Slightly more than two thirds (68.4\%) had a college/university degree.

\section{2 | Measurement}

\subsection{1 | ICD-11 PTSD and CPTSD}

The ITQ (Cloitre et al., 2015; Hyland et al., 2017c) is a self-report measure of ICD-11 PTSD and DSO symptoms and is currently under development. Hebrew Translation (Rachel Dekel and Ohad Gilbar). The author M.B. has reviewed the Hebrew translation and suggested some refinements during the translation and back translation.

The ITQ initially assesses an index trauma, how long ago this trauma occurred, and whether a person possesses a clear memory of the index trauma. With this traumatic event in mind, respondents are instructed to indicated how much they have been bothered by each symptom in the past month, using a five-point Likert scale ranging from "not at all" (0) to "extremely" (4).
There are a total of 12 PTSD symptoms included in the ITQ. Eight symptoms reflect the Re cluster, two of which are used for diagnostic purposes (Re1 Upsetting dreams, Re2 Reliving the event in the here and now). Two symptoms reflect the Av cluster (Av1 Internal reminders, Av2 External reminders), and two symptoms reflect the Th cluster (Th1 Hypervigilance, Th2 Exaggerated startle response). There are also three items that screen for functional impairment associated with these symptoms (ratings of the degree of impairment in (1) relationships and social life, (2) work or ability to work, and (3) other important aspects of life such as parenting, school/college work, or other important activities). In our sample, the internal reliability (Cronbach's alpha) of the six PTSD items used for diagnostic purposes was satisfactory ( $\alpha=0.89$ ), as were the reliabilities for the $\operatorname{Re}(\alpha=0.80), \operatorname{Av}(\alpha=0.87)$, and Th $(\alpha=0.86)$ clusters.

To assess the DSO symptoms, participants are asked to respond to a set of questions reflecting how they typically feel, think about themselves, and relate to others. The same five-point Likert scale is used for the DSO symptoms. Nine items capture the AD cluster, five of which measure hyperactivation (AD1-AD5) (e.g., When I am upset, it takes me a long time to calm down) and four measure hypoactivation (AD6-AD9) (e.g., I feel numb or emotionally shut down). Four questions capture the NSC cluster (NSC1-NSC4) (e.g., I often feel ashamed of myself whether it makes sense or not), and three questions capture the DR cluster (DR1DR3) (e.g., I feel distant or cut off from people). As with the PTSD symptoms, there are three items that screen for functional impairment associated with these symptoms. In our sample, the internal reliability of the 16 DSO items was satisfactory $(\alpha=0.94)$, as were the reliability estimates for the $\operatorname{AD}(\alpha=0.88)$, hyperactivation ( $\alpha=0.82$ ), hypoactivation $(\alpha=0.81)$, NSC ( $\alpha=0.93)$, and DR $(\alpha=0.91)$ clusters.

Current ITQ thresholds specify a score of $\geq 2$ (moderately) for at least one of the two symptoms from each of the Re, $A v$, and Th clusters. The thresholds for the DSO clusters specify the following: A score of $\geq 10$ for items AD1-AD5 or a score of $\geq 8$ for items AD6-AD9; a score $\geq 8$ for NSC1-NSC4; and a score $\geq 6$ for DR1-DR3. Diagnosis of CPTSD also requires endorsement of functional impairment. Based on the ICD-11 taxonomic structure, a person may only receive a diagnosis of PTSD or CPTSD, but not both.

\subsection{2 | Lifetime traumatic exposure}

The Life Events Checklist (LEC; Gray, Litz, Hsu, \& Lombardo, 2004) revised version (LEC-R). The LEC-R is a 19-item self-report measure designed to screen for potentially traumatic events in a respondent's lifetime. The LEC-R assesses exposure to 18 traumatic events (e.g., natural disaster, physical assault, life-threatening illness/injury), two of which specifically inquire about childhood trauma (i.e., childhood sexual abuse, childhood physical abuse) and the 19th item, "any other very stressful event/experience," can be used to indicate exposure to a trauma that is not listed. For each item, respondents check whether the event (1) "happened to me," (2) "witnessed it happening to somebody else," (3) "learned about it happening to someone close to me," (4) "part of my job," (5) "not sure it applies," (6) "doesn't apply to my experience." Each item was recoded as (1) "happened to me" and (0) all other responses, except for the items relating to "sudden violent death" and 
"sudden accidental death" that were coded (1) "witnessed it happening to somebody else" and (0) all other responses. A summed total of all binary responses was calculated to represent the number of different life events that has been experienced, this produced a single "total traumas" variable with possible scores ranging from 0 to 19.

Psychological wellbeing was assessed using the five-item World Health Organization Well-Being Index (WHO-5; World Health Organization: Regional Office for Europe, 1998). The WHO-5 is a widely used, internationally validated measure of positive mental health. A recent review of 213 international studies supported the reliability and validity of the scale (Topp, Østergaard, Søndergaard, \& Bech, 2015). Respondents are asked to indicate how they have been feeling over the past 2 weeks to each positively phrased statement along a six-point Likert scale ranging from "at no time" (0) to "all of the time" (5). Scores range from 0 to 25 with higher scores reflecting greater psychological wellbeing. Scores $\leq 13$ are indicative of poor mental health and the possible presence of a psychiatric disorder (Awata et al., 2007). The reliability of the WHO-5 among the current sample was satisfactory $(\alpha=0.93)$.

\section{3 | Statistical analysis}

The analytic plan for the current study included three phases. Phase 1 involved estimating prevalence of PTSD and CPTSD and assessing if there was a difference between males and females. The associations between diagnostic status and demographic and trauma factors were also assessed. Phase 2 involved testing six-factor analytic models shown in Figure 1.

Model 1 is a one-factor model where all symptoms load on the single latent variable CPTSD. Model 2 is a correlated first-order six-factor model (Re, Av, Th, AD, NSC, and DR). Model 3 replaced the single $A D$ latent variable with two latent variables, with five symptoms (AD1-AD5) measuring hyperactivation $(\mathrm{Hr})$ and four (AD6-AD9) measuring hypoactivation (Ho). Model 4 tests the hypothesis that the covariation among the six first-order factors can be explained by a single second-order factor (CPTSD). Model 5 specified two correlated second-order factors (PTSD and DSO) to explain the covariation among the six first-order factors; Re, Av, and Th loaded on the PTSD factor and AD, NSC, and DR loaded on the DSO factor. Model 6 was similar to Model 5 but separated the AD symptoms into the $\mathrm{Hr}$ and $\mathrm{Ho}$ latent variables. For all models the error variances were uncorrelated. Each model was specified using robust maximum-likelihood estimation (Yuan \& Bentler, 2000), which has been shown to produce correct parameter estimates, standard errors, and test statistics (Rhemtulla, Brosseau-Liard, \& Savalei, 2012). Goodness of fit for each model was assessed with a range of fit indices including the chi square, the comparative fit index (CFI; Bentler, 1990), and the Tucker-Lewis index (TLI; Tucker \& Lewis, 1973). A nonsignificant $\chi^{2}$ and values greater than 0.90 for the CFI and TLI were considered to reflect acceptable model fit. Additionally, the root mean square error of approximation (RMSEA; Steiger, 1990) was reported, where a value less than 0.05 indicated close fit and values up to 0.08 indicated reasonable errors of approximation (Jöreskog \& Sörbom, 1993). The same cut-off values can be used for the standardized root mean square residual (SRMR;
Jöreskog \& Sörbom, 1981). The Bayesian Information Criterion (BIC; Schwartz, 1978) was also used to assess the relative fit of the models. The model with the lowest BIC was considered to be the better model, and a difference greater than 10 was considered to be indicative of a "significant" difference (Raftery, 1995).

In Phase 3 an LCA was performed to determine the appropriate number of classes based on the probability of meeting the diagnostic thresholds for the three PTSD symptom clusters (Re, Av, and Th) and the four DSO symptom clusters (Hy, Ho, NSC, and DR). Six latent class models were assessed (one through six classes) to determine optimal fit. Age and gender were included as covariates in the models. The robust maximum likelihood estimator (Yuan \& Bentler, 2000) was used, and models were estimated using all available information. To avoid solutions based on local maxima, 500 random sets of starting values were used initially, followed by 50 final stage optimizations. The relative fit of the models was compared by using three information theory based fit statistics: The Akaike Information Criterion (AIC; Akaike, 1987), the BIC (Schwartz, 1978), and the sample size adjusted BIC (ssaBIC; Sclove, 1987). The class solution that possesses the lowest value can be judged the best model. Evidence from simulation studies have indicated that the $\mathrm{BIC}$ was the best information criterion for identifying the correct number of classes (Nylund, Asparouhov, \& Muthén, 2007). In addition, the Lo-Mendell-Rubin adjusted likelihood ratio test (LMR-A; Lo, Mendell, \& Rubin, 2001) was used to compare models with increasing numbers of latent classes. When a nonsignificant value $(P>.05)$ occurs, this suggests that the model with one less class should be accepted. Analyses in Phases 2 and 3 were conducted using Mplus 7.11 (Muthén \& Muthén, 2013).

\section{3 | RESULTS}

\subsection{Phase 1: prevalence and correlates}

The rates of endorsement at the symptom cluster level are reported in Table 1 . Significantly more females than males met the diagnostic criteria for the Re, Av, Th, and $\mathrm{Hr}$ symptom clusters.

The estimated prevalence of PTSD was $9.0 \%(n=90)$ and of CPTSD was $2.6 \%(n=26)$. There was a significant gender difference for PTSD (male $=7.0 \%$, female $=10.8 \% ; \chi^{2}(1)=4.35, P=.037$ ) but not for CPTSD (male $=3.1 \%$, female $=2.1 \% ; \chi^{2}(1)=0.95, P=.239$ ). The mean age of the CPTSD (34.46 years, SD $=12.77$ ), PTSD (37.60 years, $S D=14.51$ ), and no-diagnosis (41.05 years, $S D=14.51$ ) groups was significantly different $(F(2,1002)=4.692, P=.009)$ and post hoc tests (least significant difference) showed that the mean age of the CPTSD and PTSD groups did not differ but both were significantly younger than the no-diagnosis group $(P<.05)$. There was no association between diagnostic status (CPTSD, PTSD, no diagnosis) and area of residence (urban, rural: $\chi^{2}(2)=4.449, P=.108$ ), employment status (not in employment but seeking work, not in employment and not seeking work, full-time employed, part-time employed: $\chi^{2}(6)=8.787$, $P=.186$ ), and education (unfinished obligatory school, obligatory school level, finished high/secondary school, college/university: $\chi^{2}$ $(6)=3.842, P=.689$ ). There was a significant relationship between 


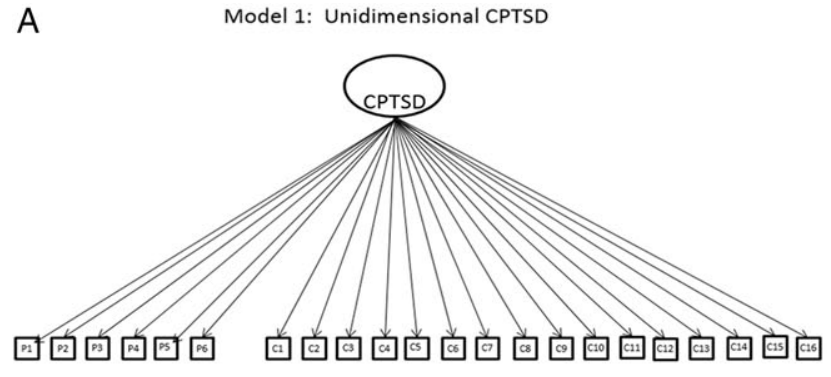

B

Model 2: 6 factor first-order model of CPTSD

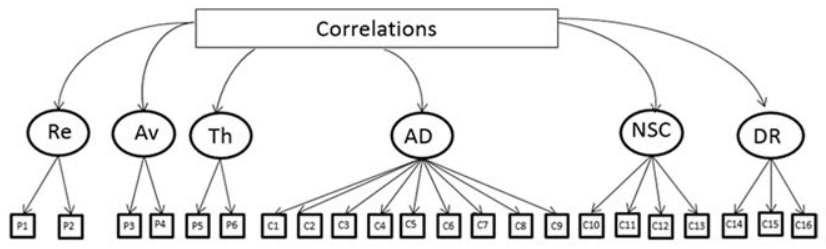

C

Model 3: 7 factor first-order model of CPTSD with affective dysregulation as two factors

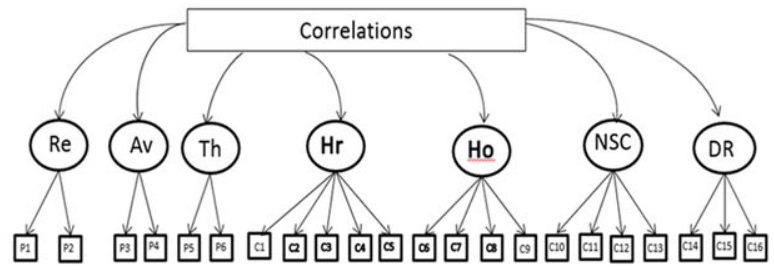

D

Model 4: Single- factor second-order with six first order factors

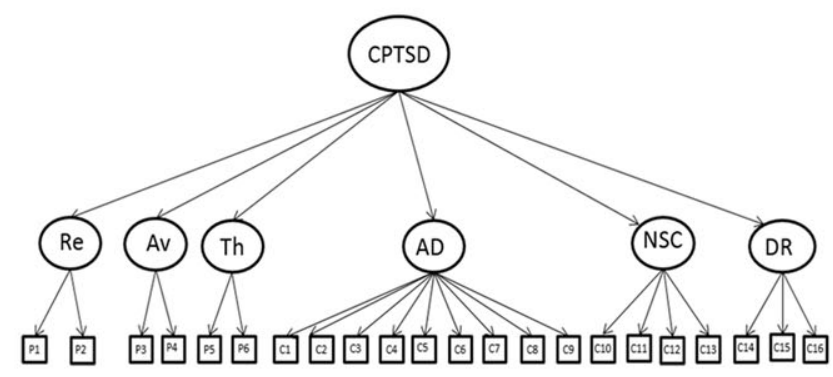

E

$\mathrm{F}$

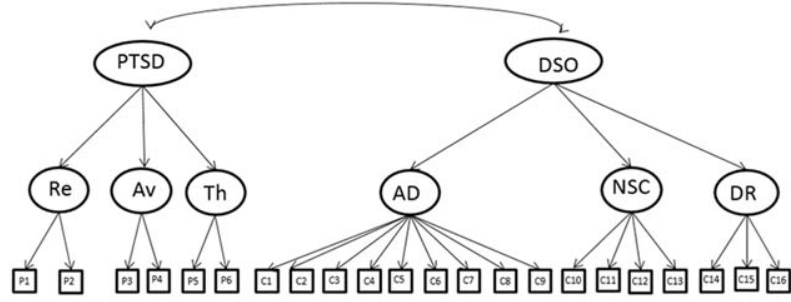

Model 6: Two-factor second-order model with affective dysregulation as two factors

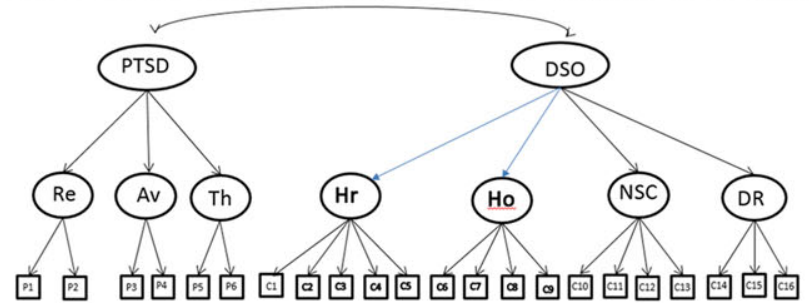

FIGURE 1 Alternative CFA models

TAB LE 1 Rates of endorsement of each PTSD and DSO symptom cluster

\begin{tabular}{|c|c|c|c|c|}
\hline & Total & Male & Female & $\chi^{2}(\mathrm{df}) P$ \\
\hline Re-experiencing & $246(24.5 \%)$ & $105(21.7 \%)$ & $141(27.2 \%)$ & 4.05 (1) .040 \\
\hline Avoidance & $289(28.8 \%)$ & $118(24.4 \%)$ & $171(32.9 \%)$ & 8.96 (1) .003 \\
\hline Hyperactivation & 201 (20.0\%) & 64 (13.2\%) & $137(26.4 \%)$ & $27.125(1)<.001$ \\
\hline Hypoactivation & $84(8.4 \%)$ & $40(8.3 \%)$ & 44 (8.5\%) & 0.015 (1).903 \\
\hline Disturbed relationships & $132(13.2 \%)$ & $62(12.8 \%)$ & $70(13.5 \%)$ & $0.101(1) .751$ \\
\hline
\end{tabular}

diagnostic status and relationship status (in a committed relationship, not in a committed relationship) with fewer participants than expected by chance with a diagnosis of CPTSD and also "in a committed relationship" (standardized adjusted residual $=-3.2$ ).

The associations between diagnostic status and exposure to different categories of trauma, as measured by the LEC, are presented in Table 2.

The associations between childhood physical abuse, physical assault, sexual assault, other unwanted or uncomfortable sexual experience, severe human suffering, and any other stressful event or experience were significantly associated with diagnostic status, and higher for the CPTSD group. Rates of childhood sexual abuse or molestation and witnessing sudden accident were significantly associated with diagnostic status, and higher for the PTSD group. The mean total number of traumas differed significantly $(F(2,1002)=9.032$, $P<.001)$ across the CPTSD (4.23, SD = 3.55), PTSD (3.72, SD = 2.45), and no-diagnosis group (2.81, SD $=2.46$ ). Post hoc tests (LSD) showed that mean number of traumas for the CPTSD and PTSD groups were not significantly different but both were significantly higher than the no-diagnosis group $(P<.05)$.

The mean WHO-5 scores differed significantly $(F(2,1002)=13.52$, $P<.001)$ across the CPTSD (10.34, SD = 6.56), PTSD (11.43, $S D=4.83)$, and no-diagnosis group (14.29, $S D=6.28)$. Post hoc tests (LSD) showed that WHO-5 scores for the CPTSD and PTSD groups were not significantly different but both were significantly lower than the no-diagnosis group $(P<.05)$. The WHO- 5 scores for the CPTSD and PTSD groups were lower than 13 , which is indicative of poor mental health. 
TABLE 2 Rates of exposure to different traumas by diagnostic classification

\begin{tabular}{|c|c|c|c|c|}
\hline & CPTSD & PTSD & No Diagnosis & $\chi^{2}(\mathrm{df}) P$ \\
\hline 1. Natural disaster & $4(15.4 \%)$ & $18(20.0 \%)$ & $128(14.4 \%)$ & $1.99(2) .369$ \\
\hline 2. Fire or explosion & $2(7.7 \%)$ & $7(7.8 \%)$ & $104(11.7 \%)$ & $1.61(2) .446$ \\
\hline 3. Transportation accident & $9(34.6 \%)$ & $37(41.1 \%)$ & $339(38.2 \%)$ & $0.45(2) .799$ \\
\hline 4. Serious accident & $4(15.4 \%)$ & $14(15.6 \%)$ & $130(14.7 \%)$ & $0.06(2) .970$ \\
\hline 5. Exposure to toxic substance & $2(7.7 \%)$ & $7(7.8 \%)$ & $59(6.7 \%)$ & $0.20(2) .905$ \\
\hline 6. Childhood physical abuse & $5(19.2 \%)$ & $10(11.1 \%)$ & $51(5.7 \%)$ & $10.77(2) .005$ \\
\hline 7. Physical assault as adult & $15(57.7 \%)$ & $40(44.4 \%)$ & 309 (34.8\%) & $8.55(2) .014$ \\
\hline 8. Assault with a weapon & $3(11.5 \%)$ & $4(4.4 \%)$ & $53(6.0 \%)$ & $1.80(2) .405$ \\
\hline 9. Childhood sexual abuse or molestation & $5(19.2 \%)$ & $22(24.4 \%)$ & $101(12.4 \%)$ & 10.75 (2) .005 \\
\hline 10. Sexual assault as adult & $5(19.2 \%)$ & $10(11.1 \%)$ & $56(6.3 \%)$ & $8.85(2) .012$ \\
\hline 11. Other unwanted or uncomfortable sexual experience & $10(38.5 \%)$ & $29(32.3 \%)$ & $175(19.7 \%)$ & $12.26(2) .002$ \\
\hline 12. Combat or exposure to a war zone & $8(30.8 \%)$ & $33(36.7 \%)$ & $300(33.8 \%)$ & $0.42(2) .811$ \\
\hline 13. Captivity & $0(0 \%)$ & $0(0 \%)$ & $4(0.05 \%)$ & $0.56(2) .769$ \\
\hline 14. Life-threatening illness or injury & $5(19.2 \%)$ & $12(13.3 \%)$ & $99(11.2 \%)$ & $1.91(2) .385$ \\
\hline 15. Severe human suffering & $6(23.1 \%)$ & $6(6.7 \%)$ & $48(5.4 \%)$ & $14.10(2) .001$ \\
\hline 16. Witness sudden violent death & $5(19.2 \%)$ & $12(13.3 \%)$ & $92(10.4 \%)$ & $2.66(2) .264$ \\
\hline 17. Witness sudden accidental death & $9(34.6 \%)$ & $35(38.9 \%)$ & $221(24.9 \%)$ & $9.12(2) .010$ \\
\hline 18. Serious injury, harm, or death you caused to someone else & $1(3.8 \%)$ & $3(3.3 \%)$ & $11(1.2 \%)$ & $3.41(2) .180$ \\
\hline 19. Any other very stressful event or experience & $12(46.2 \%)$ & $36(40 \%)$ & $207(23.3 \%)$ & $18.01(2) .000$ \\
\hline
\end{tabular}

TA B LE 3 Fit statistics for the alternative models of the ICD-11 PTSD and CPTSD symptoms

\begin{tabular}{lllllll} 
Model & Chi-square (df) & RMSEA $(90 \%$ CI) & CFI & TLI & SRMR & BIC \\
\hline 1 & $3,275.047(209)^{*}$ & $0.121(0.117-0.125)$ & 0.661 & 0.626 & 0.100 & $5,4257.403$ \\
2 & $1,104.959(194)^{*}$ & $0.068(0.065-0.072)$ & 0.899 & 0.880 & 0.054 & $50,903.796$ \\
3 & $824.535(188)^{*}$ & $0.058(0.054-0.062)$ & 0.930 & 0.914 & 0.053 & $5,0512.435$ \\
4 & $1,572.554(203)^{*}$ & $0.082(0.078-0.086)$ & 0.849 & 0.828 & 0.081 & $51,562.911$ \\
\hline 5 & $1,201.461(202)^{*}$ & $0.070(0.066-0.074)$ & 0.890 & 0.874 & 0.063 & $50,992.369$ \\
6 & $936.780(201)^{*}$ & $0.060(0.057-0.064)$ & 0.919 & 0.907 & 0.063 & $50,593.316$ \\
\hline
\end{tabular}

Note: ${ }^{*} P<.05 ; \mathrm{df}=$ degrees of freedom; $\mathrm{CFI}=$ comparative fit index; TLI = Tucker-Lewis index; RMSEA = root mean square error of approximation; $\mathrm{SRMR}=$ standardized root mean residual; $\mathrm{BIC}=$ Bayesian Information Criterion.

\section{2 | Phase 2: CFA results}

The fit statistics for the six models of PTSD and CPTSD are presented in Table 3.

Only models 3 and 6 met all the criteria for acceptable model fit and had the lowest values for the BIC. The chi-square statistics were statistically significant but this should not lead to the rejection of the models as the power of the chi-square test is positively related to sample size (Tanaka, 1987). Although both models have acceptable fit, Model 3 should be preferred as it had the lower BIC and the difference between the models $(\triangle \mathrm{BIC}=80.881)$ was greater than 10 , which is considered to be indicative of a "significant" difference. The standardized factor loadings and factor correlations are reported in Table S1 (see online Supporting Material).

\section{3 | Phase 3: LCA results}

Table S2 (see online Supporting Material) shows the fit indices for the LCA models with 1-6 classes. The fit indices favored a four-class solution as the BIC and SsaBIC values were lowest for this model, and the LRT-A became nonsignificant for the five-class solution. The AIC was also lowest for the four and five class solutions, so the four class solution should be preferred on the basis of parsimony. Class 1 (7.1\%, $n=71$ ) was characterized by high probabilities of meeting the diagnostic threshold for each of the PTSD and DSO symptom clusters. This class was labeled the "CPTSD class." Class 2 was the smallest class $(3.1 \%, n=31)$ and was characterized by relatively low probabilities of meeting the diagnostic threshold for the three PTSD symptom clusters, and higher probabilities of meeting the threshold for the four DSO symptom clusters. This class was labeled the "DSO only class." Class $3(65.8 \%, n=660)$ was characterized by low probabilities of meeting the threshold for all PTSD and DSO symptom clusters. This class was labeled the "Baseline class." Class $4(24 \%, n=241)$ was characterized by high probabilities of meeting the diagnostic criteria for each PTSD symptom cluster, and lower probabilities of meeting the diagnostic criteria for the DSO symptom clusters This class was labeled the "PTSD class" (see Figure 2). 


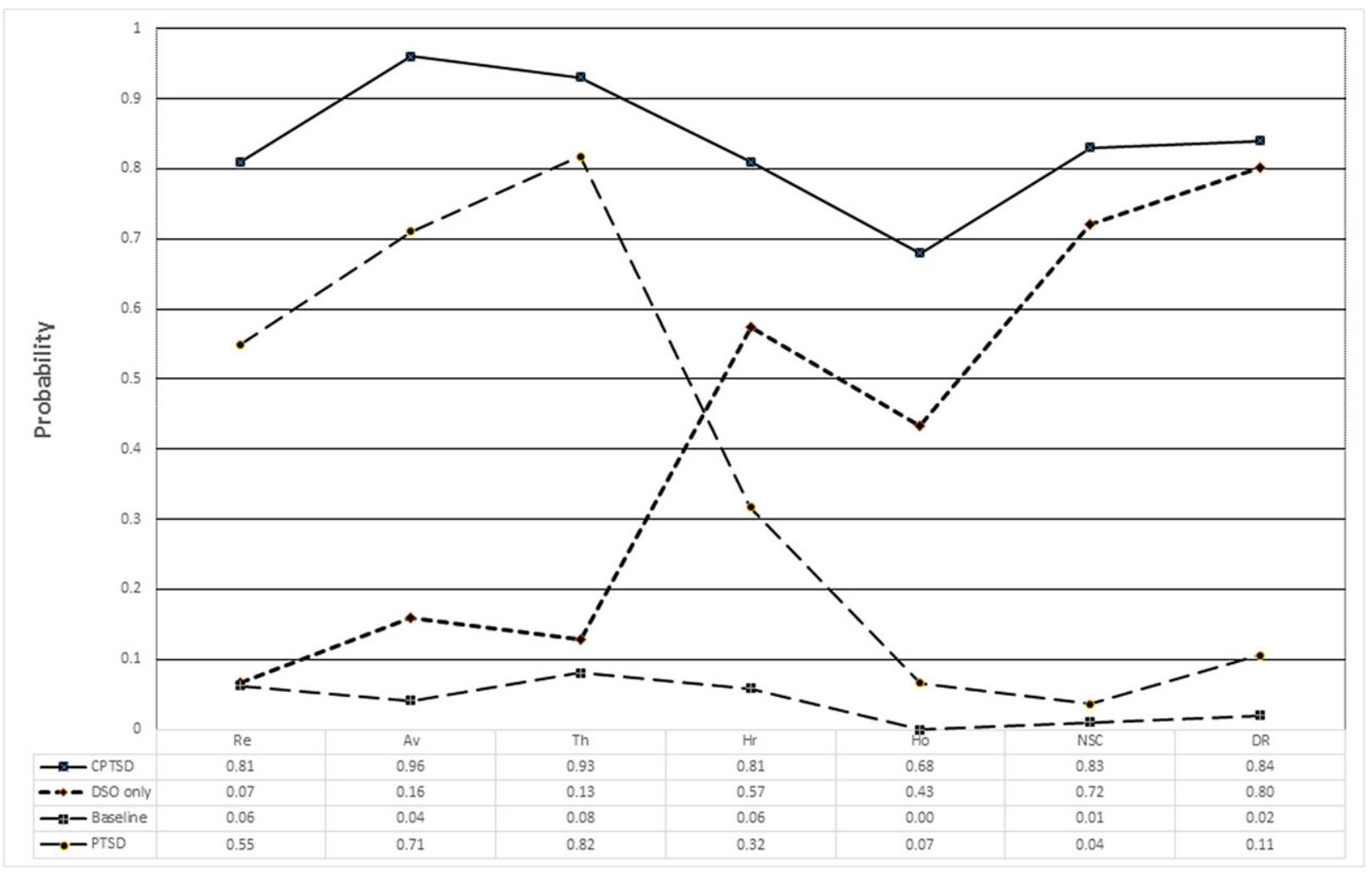

FIGURE 2 Four-class LCA profile plot

\section{4 | DISCUSSION}

This study was the first to report on the prevalence of ICD-11 PTSD and CPTSD within a nationally representative sample, using a disorderspecific measure. Additionally, the current study sought to advance the existing literature by providing the first assessment of the factorial and discriminant validity of the ICD-11 proposals for PTSD and CPTSD within a nationally representative sample.

Estimated lifetime prevalence rates of PTSD and CPTSD among the Israeli general population were 9.0 and $2.6 \%$, respectively. The combined prevalence rate of ICD-11 PTSD and CPTSD (11.6\%) in the current study is slightly higher than the $9.4 \%$ population prevalence rate reported in two previous nationally representative studies of the Israeli population (Bleich, Gelkopf, \& Solomon, 2003, Bleich, Gelkopf Melamed, \& Solomon, 2006). Variation in prevalence rates between the current and previous studies may be attributable to the use of the Diagnostic and Statistical Manual of Mental Disorders, Fourth Edition (DSM-IV; American Psychiatric Association, 1994) criteria for PTSD in the two previous assessments. The higher prevalence rate of $11.6 \%$ could also be attributed to the fact that two distinct conditions have been assessed in the present sample. However, it is also important to understand that the current study was conducted during a period of elevated terror threat along with the introduction of deliberate ignition of wildfires and urban fires as a means of terror that had a direct threat on the population.

The current results indicate that ICD-11 PTSD is more common in the general population as compared to CPTSD. This is consistent with findings from (Hyland et al., 2017b) who reported PTSD and CPTSD rates of 3.0 and $1.0 \%$, respectively, among a representative sample of Danes who were all aged 24. The higher prevalence of PTSD, relative to CPTSD, among community samples is in contrast to what has been observed among clinical samples. In a Danish treatment-seeking sample of sexual assault survivors, the prevalence of PTSD was $7.8 \%$ and the prevalence of CPTSD was $42.8 \%$ (Hyland et al., 2017a). Rates of CPTSD were also substantially higher in treatment-seeking samples from Scotland (PTSD $=37.0 \%$, CPTSD $=53.1 \%$; Karatzias et al., 2016), Wales (PTSD = 10.9\%, CPTSD = 53.6\%; Hyland et al., 2017c), and international refugees $(\mathrm{PTSD}=19.7 \%, \mathrm{CPTSD}=32.8 \%$; Nickerson et al., 2016). Initial empirical evidence suggests that while PTSD may be more common than CPTSD in the general population, complex traumatic responses are more commonly observed within clinical populations.

Females were significantly more likely than males to be diagnosed with PTSD, and no significant differences were observed in relation to CPTSD. Previous studies with clinical (Karatzias et al., 2016, 2017) and community (Hyland et al., 2017b) samples have indicated that females are approximately twice as likely as males to meet diagnostic status for ICD-11 PTSD and CPTSD; findings that are consistent with the wider trauma literature (Christiansen \& Elklit, 2012; Palic et al., 2016). Current results indicate that, among the general adult Israeli population, a meaningful gender difference exists for PTSD but there is no meaningful distinction with regards to CPTSD. It is impossible to ascertain based on the current results if the absence of any gender difference for CPTSD is a true reflection of gender differences in the wider global general population, or if the null effect is unique to the Israeli context. Taking a broader perspective shows two important differences 
between the results of our study and those of previous studies: First, in comparison to the current study, previous studies on CPTSD were conducted on specific populations such as women being treated after child abuse and children and adolescences treated for trauma, clinical community sample, individuals seeking treatment due to interpersonal trauma (Hyland et al., 2017b), and people who underwent institutional abuse as children (Knefel \& Lueger-Schuster, 2013; Knefel et al., 2015). Second, the number of participants from the general population that experienced childhood trauma was quite small and this may have affected the results. For example, only five participants met the criteria for CPTSD due to childhood physical abuse and five participants met the criteria for CPTSD due to childhood sexual abuse. Further examination of gender differences in both PTSD and CPTSD among other nationally representative samples is required, and such work is currently being undertaken in several other countries.

Prior evidence has indicated that a CPTSD diagnosis can be meaningfully distinguished from a PTSD diagnosis on the basis of several psychosocial, trauma-related, and demographic factors. In contrast to prior finding based on clinical samples, risk of CPTSD as compared to PTSD was not significantly associated with polytraumatization, psychological wellbeing, being unemployed, educational status, urbanicity, or age. CPTSD diagnosis, as compared to PTSD diagnosis, was significantly associated with not being in a committed relationship, along with exposure to unique forms of trauma including childhood physical abuse, adult physical assault, adult sexual assault (but not childhood sexual assault), other unwanted or uncomfortable sexual experiences, and exposure to severe human suffering. The conflicting results observed in the present study compared to those observed in prior studies may suggest that there are distinct risk factors for a differential diagnosis in community samples relative to clinical samples. Alternatively, the current findings may be unique to the sociocultural context of Israel and therefore point toward important cultural variations in risk for CPTSD as compared to PTSD. Further work is required on the differential predictors of CPTSD in culturally distinct community samples. Targeting cultural features in cross-cultural studies will give a better insight to the role of specific sociocultural factors in assessing PTSD and CPTSD. Furthermore, these prospective studies will enable researchers to learn about common denominators that exist crossculturally versus culture-specific factors. Testing the above will allow us to learn more about the potential contribution of specific sociocultural factors across stress disorders, namely PTSD and CPTSD, and the unique factors that will be sensitive to differentiate the two conditions.

The results from the CFA indicated that a correlated first-order model (Model 3) with three latent variables ( $R e, A v$, and Th) representing PTSD and four latent variables ( $\mathrm{Hy}, \mathrm{Ho}, \mathrm{NSC}$, and DR) representing DSO was the best fitting model. The model (Model 6) that included two second-order latent variables, PTSD and DSO, to explain the covariation among the seven first-order factors also fitted the data, but not as well as the first-order model. These results are consistent with much of the previous factor analytic work that has found that these two models are generally the best fitting models, although in clinical samples the second-order model has been found to be the best fitting model (Hyland et al., 2017a, 2017c, 2017d; Karatzias et al., 2017; Nickerson et al., 2016; Shevlin, McElroy, Bentall, Reininghaus,
\& Murphy, 2016; Tay et al., 2015). The important difference between this analysis and the previous research is that the dimensionality of the AD indicators was assessed. The results suggested that the hyperactivation and hypoactivation indicators were best represented by two correlated latent variables $(r=0.72)$ rather than one latent variable. It is clear that difficulties in emotional regulation are common consequences of trauma, particularly of an interpersonal type (Burns, Jackson, \& Harding, 2010; Ehring \& Quack, 2010), but to date the AD cluster of symptoms has been considered to be a unitary construct. More recent research has examined the role of specific facets of emotional dysregulation (Bennett, Modrowski, Kerig, \& Chaplo, 2015) and showed that there is specificity in the relationship between different types of trauma and specific types of difficulties in emotional regulation. This is important as it suggests that for a general measure of trauma response, such as the ITQ, the assessment of different aspects of emotional dysregulation (in this case hyperactivation and hypoactivation) is necessary when assessing CPTSD in populations who have been exposed to different, or multiple, forms of trauma. These results are also in line with our clinical observations in working with people with CPTSD. Furthermore, these CFA results have important implications for the ongoing work of streamlining the number of "Disturbance in Self-Organization" symptoms within the ITQ (see Shevlin et al., ). The intention is to model each DSO cluster by two items each, and these results indicate that the AD symptom cluster should comprise one "hyperactivation" and one "hypoactivation" symptom.

The results from the LCA indicated that a four class solution representing PTSD, CPTSD, DSO symptoms only, and a large baseline class was the best fitting model. This is largely consistent with the 10 studies to date that have used LCA/LPA and have generally found a distinction between symptom endorsement profiles that are representative of PTSD and CPTSD. The only study that also used a community sample (Wolf et al., 2015) reported equivocal findings where the PTSD/CPTSD distinction was evident using LCA but not when using factor mixture models. The current study, based on a large community sample, showed a clear distinction between PTSD and CPTSD. Interestingly, there was a small "DSO only class" (3.1\%) and this shows that the problems associated with DSO are not necessarily associated with the presence of PTSD symptoms. These findings are not surprising considering that DSO constructs can be cross-diagnostic phenomena. As an example, emotional dysregulation is present in anxiety and affective disorders (Hofmann, Sawyer, Fang, \& Asnaani, 2012).

Several limitations can be observed in the present study. Although it is the first to examine the nature of PTSD and CPTSD among a nationally representative adult sample, the results may not be generalizable to other nations. The unique cultural and political context of Israel, where the population lives under direct or potential threat to life means that the observed diagnostic rates may be higher than in other regions of the world (De Jong et al., 2001). Our response rate $(31 \%)$ was lower in comparison to previous study (57\%) (Bleich et al., 2003). However, the method of the two samples differs as we used Internet sampling with higher likelihood to yield lower response rates than phone surveys. Additionally, the use of a self-report method of symptom endorsement, as opposed to a clinician-administered 
diagnostic interview may too have overestimated diagnostic rates. The development of a clinician-administered diagnostic interview for ICD-11 PTSD and CPTSD is ongoing and replication across different diagnostic methods is necessary. Nevertheless, this was the first study to use a condition-specific (PTSD and CPTSD) measure in a nationally representative sample.

Overall, this study aimed to assess the prevalence of PTSD and CPTSD in a large nationally representative sample of Israeli adults; prevalence rates of PTSD and CPTSD were 9.0 and 2.6\%, respectively. The structural analyses indicated that PTSD and DSO symptom clusters were multidimensional, but not necessarily hierarchical, in nature and that there were distinct classes that were consistent with PTSD and CPTSD. These results partially support the factorial validity and more strongly support the discriminant validity of the ICD-11 proposals for PTSD and CPTSD among a community sample using a disorder-specific measure, and also support the international applicability of these diagnoses. Further research is required to determine the prevalence rates of PTSD and CPTSD in nationally representative samples across different countries and to explore the predictive utility of different types of traumatic life events for PTSD and CPTSD.

\section{ACKNOWLEDGMENT}

The study was funded by an internal research grant awarded to Professor Ben-Ezra from Ariel University RA1700000037.

\section{ORCID}

Menachem Ben-Ezra (D) http://orcid.org/0000-0002-7890-2069

\section{REFERENCES}

Akaike, H. (1987). Factor analysis and the AIC. Psychometrika, 52, 317-332.

American Psychiatric Association. (1994). Diagnostic and statistical manual of mental disorders(4th edn.). Washington, DC: Author.

Awata, S., Bech, P., Koizumi, Y., Seki, T., Kuriyama, S., Hozawa, A., ... Tsuji, I. (2007). Validity and utility of the Japanese version of the WHOFive Well-Being Index in the context of detecting suicidal ideation in elderly community residents. International Psychogeriatrics, 19, 77-88. https://doi.org/10.1017/S1041610206004212

Badour, C. L., \& Adams, T. G. (2015). Contaminated by trauma: Understanding links between self-disgust, mental contamination, and PTSD. In P. Overton, J. Simpson, \& P. Powell (Eds.), The revolting self: Psychological and clinical perspectives on self-directed disgust (pp. 127-149). London: Karnac Books.

Bennett, D. C., Modrowski, C. A., Kerig, P. K., \& Chaplo, S. D. (2015). Investigating the dissociative subtype of posttraumatic stress disorder in a sample of traumatized detained youth. Psychological Trauma, 7, 465472. https://doi.org/10.1037/tra0000057

Bentler, P. M. (1990). Comparative fit indexes in structural models. Psychological Bulletin, 107, 238-246.

Bleich, A., Gelkopf, M., Melamed, Y., \& Solomon, Z. (2006). Mental health and resiliency following 44 months of terrorism: A survey of an Israeli national representative sample. BMC Medicine, 4, 21. https://doi.org/ 10.1186/1741-7015-4-21

Bleich, A., Gelkopf, M., \& Solomon, Z. (2003). Exposure to terrorism, stress-related mental health symptoms, and coping behaviors among a nationally representative sample in Israel. Journal of the American Medical Association, 290, 612-620. https://doi.org/10.1001/jama.290.5.612

Bodas, M., Siman-Tov, M., Kreitler, S., \& Peleg, K. (2017). The role of victimization in shaping households' preparedness for armed conflicts in Israel. Disaster Medicine and Public Health Preparedness, 1-9. https://doi.org/10.1017/dmp.2017.38

Brewin, C. R., Cloitre, M., Hyland, P., Shevlin, M., Maercker, A., Bryant, R., ... Reed, G. M. (2017). Evidence regarding the ICD-11 proposals for diagnosing PTSD and complex PTSD. Clinical Psychology Review, 58, 1-15. https://doi.org/10.1016/j.cpr.2017.09.001

Burns, E. E., Jackson, J. L., \& Harding, H. G. (2010). Child maltreatment, emotion regulation, and posttraumatic stress: The impact of emotional abuse. Journal of Aggression, Maltreatment \& Trauma, 19, 801-819. https://doi.org/10.1080/10926771.2010.522947

Christiansen, D. M., \& Elklit, A. (2012). Sex differences in PTSD. In A. Lazinica \& E. Ovuga (Eds.), Posttraumatic stress disorder in a global context (pp. 113-142). Rijeka, Croatia: InTech-Open Access Book.

Cloitre, M., Garvert, D. W., Brewin, C. R., Bryant, R. A., \& Maercker, A. (2013). Evidence for proposed ICD-11 PTSD and complex PTSD: A latent profile analysis. European Journal of Psychotraumatology, 4, 20706. https:// doi.org/10.3402/ejpt.v4i0.20706

Cloitre, M., Garvert, D. W., Weiss, B., Carlson, E. B., \& Bryant, R. A. (2014). Distinguishing PTSD, complex PTSD, and borderline personality disorder: A latent class analysis. European Journal of Psychotraumatology, 5, 25097. https://doi.org/10.3402/ejpt.v5.25097

Cloitre, M., Roberts, N. P., Bisson, J. I., \& Brewin, C. R. (2015). The ICD-11 Trauma Questionnaire. Self-Report Community Version 1.0. Unpublished manuscript.

De Jong, J. T., Komproe, I. H., Van Ommeren, M., El Masri, M., Araya, M., Khaled, N., ... Somasundaram, D. (2001). Lifetime events and posttraumatic stress disorder in 4 postconflict settings. Journal of the American Medical Association, 286, 555-562. https://doi.org/10.1001/jama. 286.5.555

Dvir, Y., Ford, J. D., Hill, M., \& Frazier, J. A. (2014). Childhood maltreatment, emotional dysregulation, and psychiatric comorbidities. Harvard Review of Psychiatry, 22, 149-161. https://doi.org/10.1097/ HRP.0000000000000014

Ehring, T., \& Quack, D. (2010). Emotion regulation difficulties in trauma survivors: The role of trauma type and PTSD symptom severity. Behavior Therapy, 41, 587-598. https://doi.org/10.1016/j.beth.2010.04.004

Elklit, A., Hyland, P., \& Shevlin, M. (2014). Evidence of symptom profiles consistent with posttraumatic stress disorder and complex posttraumatic stress disorder in different trauma samples. European Journal of Psychotraumatology, 5, 24221. https://doi.org/10.3402/ejpt.v5.24221

Glück, T. M., Knefel, M., Tran, U. S., \& Lueger-Schuster, B. (2016). PTSD in ICD-10 and proposed ICD-11 in elderly with childhood trauma: Prevalence, factor structure, and symptom profiles. European Journal of Psychotraumatology, 7, 29700. https://doi.org/10.3402/ejpt.v7.29700

Gray, M. J., Litz, B. T., Hsu, J. L., \& Lombardo, T. W. (2004). Psychometric properties of the life events checklist. Assessment, 11, 330-341. https://doi.org/10.1177/1073191104269954

Hofmann, S. G., Sawyer, A. T., Fang, A., \& Asnaani, A. (2012). Emotion dysregulation model of mood and anxiety disorders. Depression and Anxiety, 29, 409-416. https://doi.org/10.1002/da.21888

Hyland, P., Brewin, C. R., \& Maercker, A. (2017d). Predictive validity of ICD11 PTSD as measured by the impact of event scale-revised: A 15-year prospective study of political prisoners. Journal of Traumatic Stress, 30, 125-132. https://doi.org/10.1002/jts.22171

Hyland, P., Murphy, J., Shevlin, M., Vallières, F., McElroy, E., Elklit, A., ... Cloitre, M. (2017b). Variation in post-traumatic response: The role of trauma type in predicting ICD-11 PTSD and CPTSD symptoms. Social 
Psychiatry and Psychiatric Epidemiology, 52, 727-736. https://doi.org/ 10.1007/s00127-017-1350-8

Hyland, P., Shevlin, M., Brewin, C. R., Cloitre, M., Downes, A. J., Jumbe, S., ... Roberts, N. P. (2017c). Validation of posttraumatic stress disorder (PTSD) and complex-PTSD using the International Trauma Questionnaire. Acta Psychiatrica Scandinavica, 136, 313-322. https://doi.org/ 10.1111/acps.12771

Hyland, P., Shevlin, M., Elklit, A., Murphy, J., Vallières, F., Garvert, D. W., \& Cloitre, M. (2017a). An assessment of the construct validity of the ICD-11 proposal for complex posttraumatic stress disorder. Psychological Trauma, 9, 1-9. https://doi.org/10.1037/tra0000114

Jöreskog, K. G., \& Sörbom, D. (1981). LISREL V: Analysis of linear structural relationships by maximum likelihood and least squares methods. Chicago: National Educational Resources.

Jöreskog, K. G., \& Sörbom, D. (1993). LISREL 8: Structural equation modeling with the SIMPLIS command language. Chicago: Scientific Software International.

Karatzias, T., Shevlin, M., Fyvie, C., Hyland, P., Efthymiadou, E., Wilson, D., ... Cloitre, M. (2016). An initial psychometric assessment of an ICD-11 based measure of PTSD and complex PTSD (ICD-TQ): Evidence of construct validity. Journal of Anxiety Disorders, 44, 73-79. https://doi.org/ 10.1016/j.janxdis.2016.10.009

Karatzias, T., Shevlin, M., Fyvie, C., Hyland, P., Efthymiadou, E., Wilson, D., ... Cloitre, M. (2017). Evidence of distinct profiles of posttraumatic stress disorder (PTSD) and complex posttraumatic stress disorder (CPTSD) based on the new ICD-11 Trauma Questionnaire (ICD-TQ). Journal of Affective Disorder, 207, 181-187. https://doi.org/ 10.1016/j.jad.2016.09.032

Knefel, M., Garvert, D. W., Cloitre, M., \& Lueger-Schuster, B. (2015). Update to an evaluation of ICD-11 PTSD and complex PTSD criteria in a sample of adult survivors of childhood institutional abuse by Knefel \& Lueger-Schuster (2013): A latent profile analysis. European Journal of Psychotraumatology, 6, 25290. https://doi.org/10.3402/ejpt.v6. 25290

Knefel, M., \& Lueger-Schuster, B. (2013). An evaluation of ICD-11 PTSD and complex PTSD criteria in a sample of adult survivors of childhood institutional abuse. European Journal of Psychotraumatology, 4, 22608. https://doi.org/10.3402/ejpt.v4i0.22608

Lo, Y., Mendell, N. R., \& Rubin, D. B. (2001). Testing the number of components in a normal mixture. Biometrika, 88, 767-778. https://doi.org/ 10.1093/biomet/88.3.767

Maercker, A., Brewin, C. R., Bryant, R. A., Cloitre, M., Reed, G. M., van Ommeren, M., ... Saxena, S. (2013). Proposals for mental disorders specifically associated with stress in the ICD-11. Lancet, 381, 16831685. https://doi.org/10.1016/S0140-6736(12)62191-6

Murphy, S., Elklit, A., Dokkedahl, S., \& Shevlin, M. (2016). Testing the validity of the proposed ICD-11 PTSD and complex PTSD criteria using a sample from Northern Uganda. European Journal of Psychotraumatology, 7, 32678. https://doi.org/10.3402/ejpt.v7.32678

Muthén, L. K., \& Muthén, B. O. (2013). Mplus 7.11. Los Angeles, CA: Muthén \& Muthén.

Nickerson, A., Cloitre, M., Bryant, R. A., Schnyder, U., Morina, N., \& Schick, M. (2016). The factor structure of complex posttraumatic stress disorder in traumatized refugees. European Journal of Psychotraumatology, 7, 33253. https://doi.org/10.3402/ejpt.v7.33253

Nylund, K. L., Asparouhov, T., \& Muthén, B. O. (2007). Deciding on the number of classes in latent class analysis and growth mixture modeling: $A$ Monte Carlo simulation study. Structural Equation Modeling, 14, 535569. https://doi.org/10.1080/10705510701575396

Palic, S., Zerach, G., Shevlin, M., Zeligman, Z., Elklit, A., \& Solomon, Z. (2016). Evidence of complex posttraumatic stress disorder (CPTSD) across populations with prolonged trauma of varying interpersonal intensity and ages of exposure. Psychiatry Research, 246, 692-699. https://doi.org/10.1016/j.psychres.2016.10.062

Perkonigg, A., Hofler, M., Cloitre, M., Wittchen, H. U., Trautmann, S., \& Maercker, A. (2016). Evidence for two different ICD-11 posttraumatic stress disorder in a community sample of adolescents and young adults. European Archives of Psychiatry and Clinical Neuroscience, 266, 317-328. https://doi.org/10.1007/s00406-015-0639-4

Raftery, A. E. (1995). Bayesian model selection in social research. Sociological Methodology, 25, 111-163. https://doi.org/10.2307/271063

Rhemtulla, M., Brosseau-Liard, P. É., \& Savalei, V. (2012). When can categorical variables be treated as continuous? A comparison of robust continuous and categorical SEM estimation methods under suboptimal conditions. Psychological Methods, 17, 354-373. https://doi.org/ 10.1037/a0029315

Sachser, C., Keller, F., \& Goldbeck, L. (2017). Complex PTSD as proposed for ICD-11: Validation of a new disorder in children and adolescents and their response to trauma-focused cognitive behavioral therapy. Journal of Child Psychology and Psychiatry, 58, 160-168. https://doi.org/ 10.1111/jcpp.12640

Schwarz, G. (1978). Estimating the dimension of a model. Annals of Statistics, 6,461-464.

Sclove, S. L. (1987). Application of model-selection criteria to some problems in multivariate analysis. Psychometrika, 52, 333-343.

Shevlin, M., Hyland, P., Karatzias, T., Fyvie, C., Roberts, N., Bisson, J. I., ... Cloitre, M. (2017). Alternative models of disorders of traumatic stress based on the new ICD-11 proposals. Acta Psychiatrica Scandinavica, 135, 419-428. https://doi.org/10.1111/acps.12695

Shevlin, M., Hyland, P., Roberts, N. P., Bisson, J. I., Brewin, C. R., \& Cloitre, M. (2018). A psychometric assessment of Disturbances in Self-Organization symptom indicators for ICD11 Complex PTSD using the International Trauma Questionnaire. European journal of psychotraumatology, 9(1), 1419749. https://doi.org/10.1080/20008198.2017.1419749

Shevlin, M., McElroy, E., Bentall, R. P., Reininghaus, U., \& Murphy, J. (2016). The psychosis continuum: Testing a bifactor model of psychosis in a general population sample. Schizophrenia Bulletin, 43, 133-141. https://doi.org/10.1093/schbul/sbw067

Steiger, J. H. (1990). Structural model evaluation and modification: An interval estimation approach. Multivariate Behavioral Research, 25, 173-180. https://doi.org/10.1207/s15327906mbr2502_4

Tanaka, J. S. (1987). How big is big enough? Sample size and goodness of fit in structural equation models with latent variables. Child Development, 58, 134-146. https://doi.org/10.2307/1130296

Tay, A. K., Mohsin, M., Rees, S., Steel, Z., Tam, N., Soares, Z., ... Silove, D. (2017). The factor structures and correlates of PTSD in post-conflict Timor-Leste: An analysis of the Harvard Trauma Questionnaire. BMC Psychiatry, 17, 191. https://doi.org/10.1186/s12888-017-1340-0

Tay, A. K., Rees, S., Chen, J., Kareth, M., \& Silove, D. (2015). The structure of post-traumatic stress disorder and complex post-traumatic stress disorder amongst West Papuan refugees. BMC Psychiatry, 15, 111. https://doi.org/10.1186/s12888-015-0480-3

Topp, C. W., Østergaard, S. D., Søndergaard, S., \& Bech, P. (2015). The WHO5 Well-Being Index: A systematic review of the literature. Psychotherapy and Psychosomatics, 84, 167-176. https://doi.org/10.1159/00037 6585

Tucker, L. R., \& Lewis, C. A. (1973). Reliability coefficient for maximum likelihood factor analysis. Psychometrika, 3, 1-10.

Walsh, K., Fortier, M. A., \& DiLillo, D. (2010). Adult coping with childhood sexual abuse: $A$ theoretical and empirical review. Aggression and Violent Behaviour, 15, 1-13. https://doi.org/10.1016/j.avb.2009.06.009 
Wolf, E. J., Miller, M. W., Kilpatrick, D., Resnick, H. S., Badour, C. L, Marx, B. P., ... Friedman, M. J. (2015). ICD-11 complex PTSD in US National and Veteran Samples: Prevalence and structural associations with PTSD. Clinical Psychological Science, 3, 215-229. https://doi.org/ $10.1177 / 2167702614545480$

World Health Organization: Regional Office for Europe. (1998). Well-being measures in primary health care: The DepCare Project. Consensus Meeting, Stockholm, Sweden. Retrieved from https://www.who-5.org

Yuan, K. H., \& Bentler, P. M. (2000). Three likelihood-based methods for mean and covariance structure analysis with nonnormal missing data. Sociological Methodology, 30, 165-200. https://doi.org/10.1111/ 0081-1750.00078

\section{SUPPORTING INFORMATION}

Additional Supporting Information may be found online in the supporting information tab for this article.

How to cite this article: Ben-Ezra M, Karatzias T, Hyl P, et al. Posttraumatic stress disorder (PTSD) and complex PTSD (CPTSD) as per ICD-11 proposals: A population study in Israel. Depress Anxiety. 2018;35:264-274. https://doi.org/10.1002/ da. 22723 